\title{
Imaging Graphene by Field Ion Microscopy
}

\author{
Cédric Barroo ${ }^{1,2,3}$ and Thierry Visart de Bocarmé ${ }^{3}$
}

1. John A. Paulson School of Engineering and Applied Sciences, Harvard University, Cambridge MA USA

2. Department of Chemistry and Chemical Biology, Harvard University, Cambridge MA USA

3. Chemical Physics of Materials, Université Libre de Bruxelles (ULB), CP243, 1050 Brussels, Belgium

Since its rediscovery, isolation and characterization in 2004 [1], graphene has attracted much attention due to its exceptional properties such as optical transparency, strength, electrical and thermal conductivity. The study of graphene and other two-dimensional crystalline materials such as $\mathrm{MoS}_{2}$ and B-N structures gave rise to an abundant literature. Among the techniques used to characterize graphenebased structure, field emission techniques have been used only occasionally. Those works include the study of multiwall carbon nanotubes (MWNTs) by field ion microscopy (FIM) and field emission microscopy (FEM) to investigate the structure of the MWNTs tips and the effect of adsorption of gas molecules on the electron emission [2], as well as FIM imaging of monatomic carbon chains and the visualization of their two-dimensional wave functions [3]. Recent work also proved that these techniques can be used to study the early stages of carbon nanotube nucleation [4]. Here we propose to image CVD grown graphene by field ion microscopy.

Field emission techniques comprise the field emission (FEM) and the field ion (FIM) microscopies, which are analytical techniques based on the emission of electrons (in the case of FEM) and ions (in the case of FIM) in a projection-type microscope. These techniques use very sharp tips as samples, with a radius of curvature lower than $50 \mathrm{~nm}$, on which a high electrical field is applied to allow for electron emission in FEM or gas ionisation in FIM. With a magnification of approximately 1 million, these methods can be used to image the arrangement of surfaces or adsorbed species at the surface of the extremity of a nanosized metal tip. The typical resolution in FIM is $0.2 \mathrm{~nm}$.

In this work, graphene has been imaged by FIM at low temperature. Graphene is obtained by CVD process on a $\mathrm{Cu}$ substrate, and a polymer layer is then deposited on the graphene sheet by spin-coating. The dissolution of the copper substrate in a $\left(\mathrm{NH}_{4}\right)_{2} \mathrm{~S}_{2} \mathrm{O}_{8}$ solution results in a "floating carpet" which is optically discernible from the solution thanks to the presence of the polymer layer. The floating graphene/polymer layer is then fished with a Pt sharp tip used for FIM experiments. It has to be noted that the tip has been characterized by FIM prior to the fishing in order to be able to observe changes due to the presence of graphene. The polymer is then dissolved in ethyl acetate, and the sample is gently cleaned with isopropanol. The graphene-decorated $\mathrm{Pt}$ sample is then transferred in the microscope. Imaging conditions are: $\mathrm{T}=50 \mathrm{~K}, \mathrm{P}_{\mathrm{Ne} / \mathrm{He}}=2.1 \times 10^{-5} \mathrm{mbar}$, and the tip is positively charged $(\mathrm{F} \approx 35-$ 40 V.nm $\left.{ }^{-1}\right)$.

While the potential is increased to image the surface of the sample, two different structures can be observed: bright spots and streaked spots. Bright spots appearing at an early time of imaging usually corresponds to non-crystalline impurities at the surface of the sample and can be removed by further increase of the electrical field. These spots indeed evolve towards a crystalline structure. Streaked spots however are not usually observed in conventional FIM studies, and similar structures have been assigned to the presence of carbon nanotubes in previous work [2]. In our case, the streaked spots may 
thus correspond to the graphene sheet. While the electrical field is increased, the intensity of the streaked spots remains the same, but we can observe an increasing amount of spots, and a change of 'orientation' can be observed on some of the initial streaks. In the best case scenario, the fishing method used in this work will induce the presence of a single layer of graphene at the surface of the tip. However, there is a high probability for the graphene to fold on the tip sample. The evolution of streaked spots may thus be explained by the unfolding of the graphene sheet under a threshold value of electrical field, allowing exposing parts of graphene with different orientations, and allowing to cover a broader surface of the Pt tip (Figure 1a). Further increase in the electrical field induce the removing of the streaked spots, which is associated to the field desorption of graphene (Figure 1b).

This work shows that field ion microscopy can be used to image 2D materials by using a rather simple fishing method. The appearance of streaked spots is assigned to the presence of graphene, and it is possible to study their evolution as a function of the applied electrical field. [5]

\section{References:}

[1] K.S. Novoselov, A.K. Geim, S.V. Morozov, D. Jiang, Y. Zhang, S.V. Dubonos, I.V. Grigorieva and A.A. Firsov, Science 306 (2004) 666

[2] Y. Saito, K. Hata, A. Takakura, J. Yotani and S. Uemera, Physica B 323 (2002) 30

[3] T.I. Mazilova, I.M. Mikhailovskij, V.A. Ksenofontov and E.V. Sadanov, Nano Letters 9 (2009) 774

[4] M. Moors, H. Amara, T. Visart de Bocarmé, C. Bichara, F. Ducastelle, N. Kruse and J.-C. Charlier, ACS Nano 3 (2009) 511

[5] C.B. thanks the Fonds de la Recherche Scientifique (F.R.S.-FNRS) for financial support. The authors gratefully thank the Van-Buuren Foundation for a financial support for the acquisition of equipment.
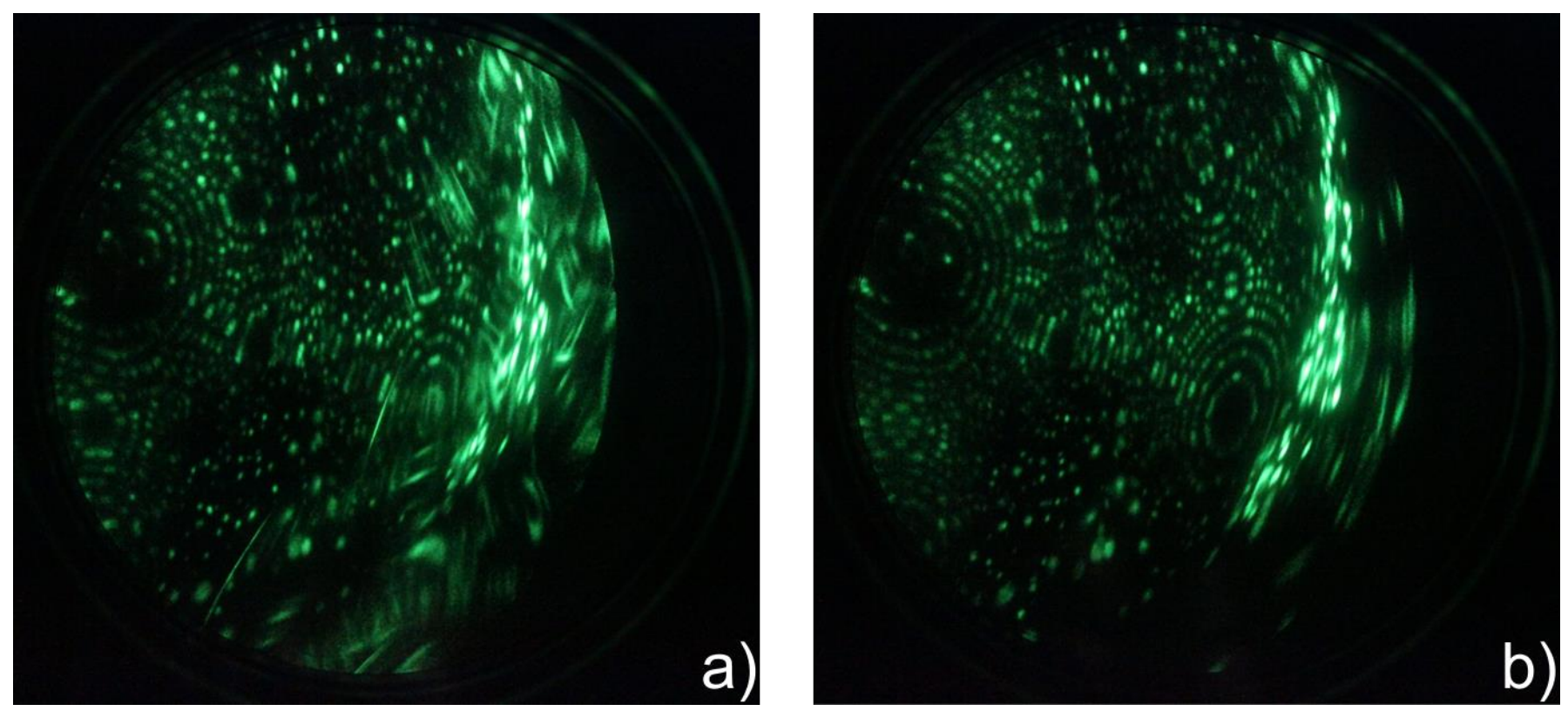

Figure 1. a) FIM imaging of a Pt tip covered with grapheme with a crystalline part (left) due to the Pt substrate, and streaked spots assigned to the presence of graphene - b) Same sample imaged with a higher imaging field showing that the graphene can be field-desorbed above a threshold value. 